\title{
Nature of Chemisorption on Titanium Carbide and Nitride
}

\author{
Carlo Ruberto * Aleksandra Vojvodic, and Bengt I. Lundqvist \\ Department of Applied Physics, Chalmers University of Technology, SE-412 96 Göteborg, Sweden
}

\begin{abstract}
Extensive density-functional calculations are performed to understand atomic chemisorption on the $\mathrm{TiC}(111)$ and $\mathrm{TiN}(111)$ surfaces, in particular the calculated pyramid-shaped trends in the adsorption energies for second- and third-period adatoms. Our previously proposed concerted-coupling model for chemisorption on $\mathrm{TiC}(111)$ is tested against new results for adsorption on $\mathrm{TiN}(111)$ and found to apply on this surface as well, thus reflecting both similarities and differences in electronic structure between the two compounds.
\end{abstract}

Keywords: Density-functional calculations, Titanium carbide, Titanium nitride, Adatoms, Adsorption, Chemisorption, Density of states.

\section{INTRODUCTION}

Within an extensive theoretical study of atomic chemisorption on the Ti-terminated polar TiX(111) (X $=\mathrm{C}, \mathrm{N}$ ) surfaces, we have presented characteristic trends in adsorption energies and geometries for $\mathrm{H}$, second-row elements B, C, N, O, and F, and third-row elements $\mathrm{Al}$, $\mathrm{Si}, \mathrm{P}, \mathrm{S}, \mathrm{Cl}$ [1, 2]. The study has both fundamental and technological motivations, as these materials are important in applications in which surface properties are essential, such as growth of alumina on $\operatorname{Ti}(\mathrm{C}, \mathrm{N})(111)$ and use of TiN as a thin diffusion barrier in integrated circuits or as a biocompatible material.

Our results show similar trends for $\mathrm{TiC}$ and $\mathrm{TiN}$, with atomic adsorption energies for the second- and thirdperiod elements varying along the periods in a characteristic pyramid-like way [Fig. 3(a)] 1, 2]. Within each period, the energies increase from group III to group VI and decrease from group VI to group VII, with overall higher values for period 2 . These variations are very large, from $\sim 3.5 \mathrm{eV}$ for $\mathrm{Al}$ to $\sim 8.5 \mathrm{eV}$ for $\mathrm{O}$. Also, the preference for adsorption on $\mathrm{TiC}$ vs. TiN varies, with a slightly stronger chemisorption on TiN for the elements toward the left of the periodic table (B and $\mathrm{Al}$ ) and a slightly stronger chemisorption on $\mathrm{TiC}$ for the remaining adatoms.

Such variations cannot be explained within a simple model, where only one type of coupling mechanism is considered. In a separate study, we propose a concertedcoupling model for adsorption on $\mathrm{TiC}(111)$, in which two different types of adatom-substrate couplings are active [1]. This is similar to the $d$-band model for chemisorption on transition metals [3], however, with very different couplings, due to the more complex electronic structure of TiC. In this contribution, we pursue our investigation further, analyzing the electronic structure of the different adsorbates on TiN(111) and discussing whether our proposed model can be generalized and used to understand the similarities and differences between the $\mathrm{TiN}(111)$ and $\mathrm{TiC}(111)$ substrates.

\footnotetext{
*Electronic address: ruberto@fy.chalmers.se
}

\section{COMPUTATIONAL METHOD}

The computational method used is described in Ref. 2]. In short, the pseudopotential-plane-wave densityfunctional-theory (DFT) code dacapo is used, with PW91 GGA exchange-correlation potential, ultrasoft pseudopotentials, and slab geometry [4].

In the present paper, the electronic structures of the considered systems are analyzed by calculating and plotting the total and local densities of states (DOS and LDOS, respectively) obtained from the calculated KohnSham (KS) wavefunctions and energy eigenvalues. Atomand/or orbital-projected $\operatorname{LDOS}(E)$ 's are obtained by projecting the KS wavefunctions onto the individual atomic orbitals and then plotting them as functions of the energy relative to the Fermi level, $E-E_{F}$. The spatial extent of the DOS's at specific energy intervals is also analyzed in detail by examining the KS-wavefunction amplitudes for the relevant electronic states in three-dimensional real space. Also, the charge localization around individual atoms is analyzed with the "atoms-in-molecule" method of Bader [5].

\section{RESULTS AND ANALYSIS}

\section{A. Electronic structure of the clean $\mathrm{TiX}(111)$ surfaces}

Bulk $\mathrm{TiC}$ and TiN adopt the $\mathrm{NaCl}$ structure. Thus, in the (111) direction, the crystals are composed of alternating $\mathrm{Ti}$ and $\mathrm{X}$ atomic layers, in an $A B C$ stacking sequence. Our study focuses on the Ti-terminated surfaces, since experiments show this to be the stable termination for $\mathrm{TiC}(111)$ [6]. The calculated lattice parameters and surface relaxations are given in Refs. 1, 2].

Both Ti-terminated TiX(111) surfaces are characterized by the presence of strong Ti-localized surface resonances (TiSR's) [7], pinned at $E_{F}$ [Figs. [1(a-b)] and consisting of mainly $d_{(x z, y z)}$ and $d_{\left(x y, x^{2}-y^{2}\right)}$ orbitals (where $z$ is perpendicular to the surface). Figure 1(c) shows this symmetry and how the TiSR electron distribution is localized mainly around the Ti atoms (as evidenced also by the small width of the TiSR peaks) and extends toward 
the fcc surface sites.

Our calculated surface DOS's [Figs. [(a-b)] show that the TiSR is more filled on $\operatorname{TiN}(111)$ than on $\mathrm{TiC}(111)$. This is confirmed by a Bader analysis of the surface charges, yielding a surface-Ti ionicity of +1.09 for $\mathrm{TiC}$ and of +0.96 for TiN. This can be understood by considering the filling of the TiSR's as the mechanism that compensates the electrostatic instability of the polar TiX(111) surfaces [8]. As bulk TiN is more ionic than bulk $\mathrm{TiC}(\mathrm{Ti} \rightarrow \mathrm{X}$ charge transfers of 1.51 electrons for $\mathrm{TiC}$ and 1.62 for TiN, according to our Bader analysis) [2], a larger compensating surface charge is needed on $\operatorname{TiN}(111)$.

The bulk electronic structure of TiX [inserts in Figs. [1(a-b)] consists of (i) a lower valence band (LVB), of only $\mathrm{X} 2 s$ character, (ii) an upper valence band (UVB), characterized by bonding Ti3 $d-\mathrm{X} 2 p$ states in its high-energy part and by $\mathrm{X} 2 p-\mathrm{X} 2 p$ bonding interactions in its lowenergy part, and (iii) a conduction band (CB), of mainly Ti3 $d$ character, corresponding to antibonding Ti3 $3-\mathrm{X} 2 p$ states [1, 2]. The ionicity of bulk TiX is evidenced by a predominance of $\mathrm{Ti}(\mathrm{X})$ character in the $\mathrm{CB}$ (UVB).

Both TiX(111)-surface UVB's [Figs. 1(a-b)] are composed of one main peak, three smaller peaks at lower energies, a shoulder on the high-energy side of the main peak, and a small peak at the upper edge of the UVB. For both surfaces, our detailed analysis of the individual KS wavefunctions composing the UVB (Fig. 2) shows that, similarly to the bulk UVB, the main UVB peak and the high-energy UVB region consist of Ti-X bonding states [Fig. 2(c)]. On the other hand, the three lowenergy peaks consist almost exclusively of $\mathrm{X}-\mathrm{X}$ bonding states that are mainly localized to the surface region [Figs. [2 $(\mathrm{a}-\mathrm{b})]$, that is, they are $\mathrm{X}$-localized surface resonances (XSR's).

Like for the bulk DOS 2], several differences exist between the TiN(111) and TiC(111) surface DOS's [Figs. 1(a-b)]: (i) the UVB and CB of TiN(111) lie at lower energies, due to the partial filling of the $\mathrm{CB}$ with the extra electron per formula unit present in TiN; (ii) the pseudogap between the UVB and the TiSR is larger for TiN(111); and (iii) the UVB DOS is considerably larger and more X-localized on TiN, which again is an indication of the higher ionicity of TiN.

Thus, both surface DOS's differ from their corresponding bulk DOS's [see Figs. प(a-b)] by (i) the presence of TiSR's around $E_{F}$, (ii) a quenching of the UVB peak lying above the main peak, and (iii) the presence of XSR's in the low-energy UVB region. These changes can be understood in terms of breakage of the Ti-X and $\mathrm{X}-\mathrm{X}$ bonds that cross the (111) plane upon cleavage of the bulk structures to create the (111) surfaces. As a consequence, the bonding and antibonding $\mathrm{Ti}-\mathrm{X}$ states, which are located in the high-energy part of the UVB and in the $\mathrm{CB}$, respectively, collapse into more atomic-like $\mathrm{X}$ and Ti orbitals. Therefore, the quenching of the highenergy UVB peak can be associated with the disappearance of $\mathrm{X}$-localized bonding $\mathrm{Ti}-\mathrm{X}$ states, while the ap- pearance of the TiSR's arises from the energetical lowering of Ti-localized antibonding Ti-X states (indeed, for both compounds, a closer inspection of the $\mathrm{CB}$ reveals the quenching of a bulk $\mathrm{CB}$ peak located right above the TiSR's). The TiSR's can thus be interpreted as dangling bonds extending toward the fcc sites (which in the bulk would be occupied by X atoms) [Fig. I(c)]. Similarly, the appearance of the XSR's in the low-energy UVB region is caused by the breakage of $\mathrm{X}-\mathrm{X}$ bonding interactions, which dominate at these energies.

\section{B. Electronic structure of fcc adatoms on TiX(111)}

In a separate study, we have proposed a model for the description of the exceptionally strong atomic chemisorption on $\mathrm{TiC}(111)$ [1]. In this concerted-coupling model, based on the Newns-Anderson (NA) model [9], the adsorption is understood to arise from the concerted action of two different types of adatom-substrate coupling: a strong adatom-TiSR coupling and weaker adatom-CSR couplings.

Briefly, the NA model for chemisorption on metal surfaces yields two limiting cases: weak and strong chemisorption, corresponding, respectively, to small and large coupling matrix elements between adatom and substrate states, compared to the substrate band width. In the weak chemisorption, the adsorbate level is shifted and broadened (if it lies in the energetical region of the substrate band). In the strong chemisorption, the adatom level is replaced by two levels that lie on each side of the substrate band. These are usually interpreted as the bonding and antibonding states of the "surface molecule" formed by the adatom and its neighboring substrate atoms. These bonding and antibonding states appear gradually, as the adatom-substrate hopping integral $h$ increases. At small $h$, the adlevel is broadened. As $h$ increases, this level broadens further and splits gradually into two separate levels [10].

Our concerted-coupling model for $\mathrm{TiC}(111)$ is based on an analysis of the difference in DOS $(\triangle \mathrm{DOS})$ before and after adsorption (thus, the $\triangle \mathrm{DOS}$ shows the appearance and quenching of states due to the adsorption of an adatom as positive and negative peaks, respectively) 1]. For all considered adatoms on $\operatorname{TiC}(111)$, our $\triangle$ DOS's show: (i) the appearance of Ti-dominated states at $\sim+1.0 \mathrm{eV}$; (ii) a strong quenching of the TiSR; (iii) the appearance of states below $E_{F}$, whose energies decrease with increasing adatom number $Z$ within each adatom period (due to the decreasing free-atom energy as $Z$ increases).

Within the NA model, these results are interpreted as evidence for a strong adatom-TiSR coupling, giving rise to antibonding and bonding states above and below, respectively, $E_{F}$. At the same time, our calculated $\triangle$ DOS's show also that the bonding adatom-TiSR level below $E_{F}$ is successively broadened and split into subpeaks as its energy approaches the middle of the substrate UVB re- 


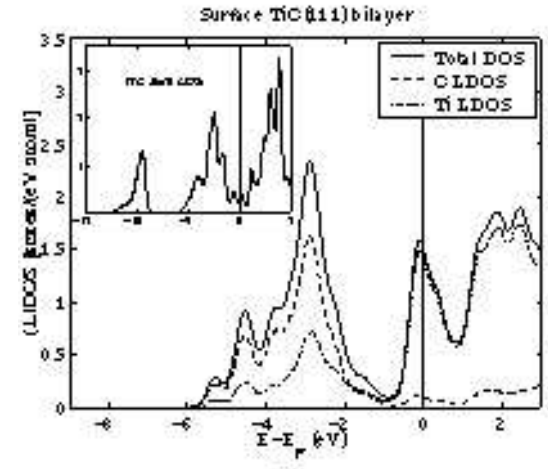

(a)

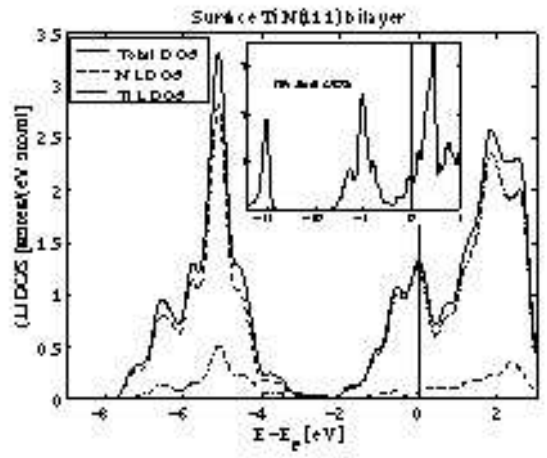

(b)

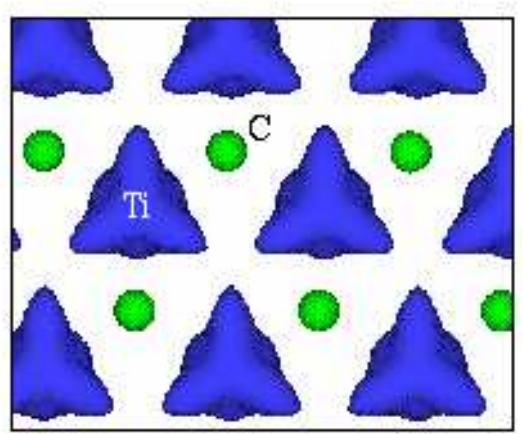

(c)

FIG. 1: (a-b) Total and atom-projected DOS for the top TiX bilayer of the clean (a) TiC(111) and (b) TiN(111) surfaces. The inserts in (a) and (b) show the total DOS for bulk TiC and TiN, respectively. (c) Real-space contour plot (from above the surface and showing only the top $\mathrm{TiC}$ bilayer) of the total charge density of the electronic states above $-0.6 \mathrm{eV}$ on $\mathrm{TiC}(111)$, showing the TiSR (C atoms: green balls; Ti atoms: inside the electron clouds, as marked in the figure).
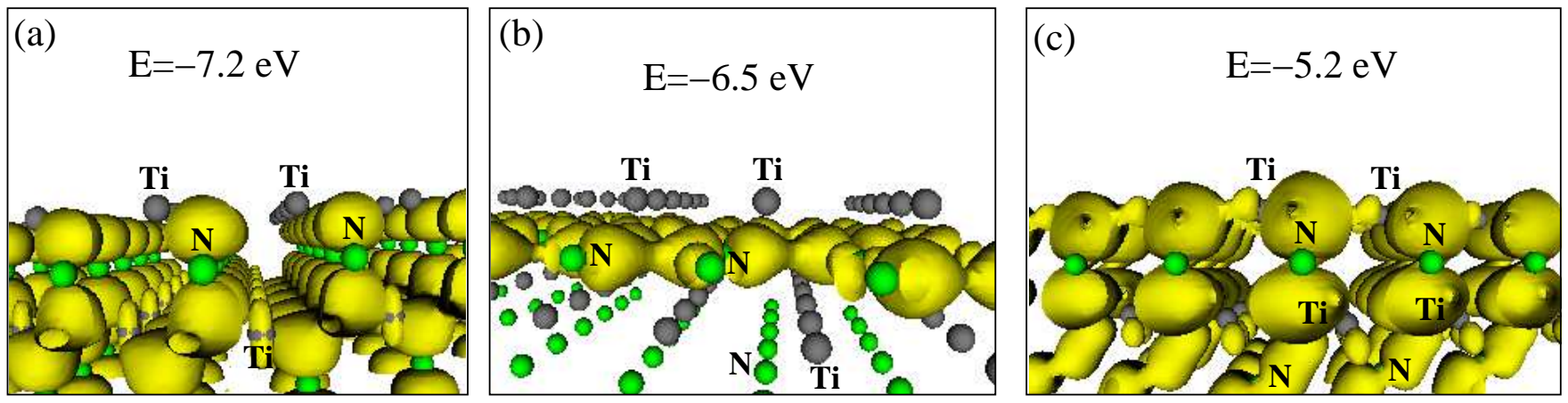

FIG. 2: Real-space contour plots of the KS wavefunctions for the clean TiN(111) surface at selected energies, viewed perpendicularly to the surface, illustrating: ( $\mathrm{a}-\mathrm{b}$ ) typical bonding $\mathrm{N}-\mathrm{N}$ interactions, with and without coupling to bulk states, in the lower UVB region; (c) a typical Ti-N bonding state, with high N-localization, in the main UVB peak. Ti (N) atoms are grey (green) balls, or lie inside the electron clouds, as marked in the figures.

gion. This indicates, within the NA model, an additional, weaker, coupling between the TiSR-modified adlevel and the substrate UVB peaks, which are characterized by CSR's. Thus, the subpeaks correspond to combinations of the bonding and antibonding solutions that arise from adatom-CSR couplings. This is confirmed by (i) the presence, in the substrate-projected $\triangle D O S$ 's, of negative peaks that are almost exclusively localized around the $\mathrm{C}$ atoms and that lie at energies in between the subpeaks and (ii) our state-resolved, real-space, analysis of the DOS, which shows that the subpeaks are dominated by adatom- $\mathrm{C}$ bonding states at lower UVB energies and by adatom-Ti bonding states at higher energies. The adatom-C states are only present at energies lower than the main substrate UVB peak and can only be interpreted as bonding solutions of adatom-CSR couplings. This analysis shows also that the adatom- $\mathrm{C}$ bonding states get successively stronger as the energetical overlap between the TiSR-modified adlevel and the substrate
CSR's increases. Maximum overlap is achieved for the $\mathrm{O}$ adatom, for which all bonding adatom-TiSR states lie in the lower half of the substrate UVB [1].

Our new calculations for atomic adsorption on TiN(111) show that the $\Delta$ DOS's [Figs. 3(b)-(k)] behave in a similar way on this substrate. Figure 4 illustrates schematically the concerted-coupling model for TiN(111), using the calculated $\triangle \mathrm{DOS}$ for fcc $\mathrm{H}$ on TiN(111), which provides the most complete illustration of the different types of adatom-substrate couplings. As can be seen, the results for adsorption on $\mathrm{TiN}(111)$ show strong similarities to those described above for $\mathrm{TiC}(111)$. However, a closer comparison reveals differences: (i) the antibonding peak above $E_{F}$ lies at a lower energy than on $\mathrm{TiC}(111),+0.4 \mathrm{eV}$; (ii) the TiSR quenching is stronger than on $\mathrm{TiC}(111)$ and involves a larger number of TiSR electrons; (iii) the coupling between the TiSR-modified adlevel and the XSR's occurs at lower energies than on $\mathrm{TiC}(111)$. Points (i) and (ii) can be understood from the 
facts that the TiN(111) TiSR lies at a lower energy than on $\mathrm{TiC}(111)$ and that this causes a larger filling of the TiSR on TiN than on TiC. Point (iii) is caused by the lower energy of the TiN(111) UVB, compared to that of the $\mathrm{TiC}(111) \mathrm{UVB}$.

In addition, our state-resolved, real-space, analysis of the DOS shows that also on $\operatorname{TiN}(111)$ there is a successively increasing dominance of adatom-X bonding states [Fig. 3(1)] with decreasing energy. Again, however, differences can be detected: (i) compared to the adatom-Ti coupling, the adatom-X coupling is generally stronger on $\mathrm{TiN}(111)$ than on $\mathrm{TiC}(111)$; (ii) while no adatom-Ti coupling can be detected in the TiSR energetical region on $\mathrm{TiC}(111)$, such adatom-Ti bonding states are found in the TiSR energetical region on TiN(111). Point (i) can be understood from the higher ionicity of TiN, which results in a stronger LDOS on the $\mathrm{N}$ atoms, compared to the $\mathrm{C}$ LDOS in TiC, and thus a stronger adatom-X overlap on $\mathrm{TiN}(111)$ than on $\mathrm{TiC}(111)$. Point (ii) can be interpreted as the presence of a weak coupling, similar to the adatom-UVB coupling, between the Ti-localized $\mathrm{CB}$ and the antibonding adatom-TiSR states. Due to the $\mathrm{CB}$ being partially filled in TiN, this adatom-CB coupling results here in filled states, in contrast to $\mathrm{TiC}$, where the CB is empty.

Our results show thus that the atomic chemisorption on $\mathrm{TiN}(111)$ can be understood and analyzed in a way similar to that on $\mathrm{TiC}(111)$. Both types of interaction of our concerted-coupling model (adatom-TiSR and adatom-XSR's) appear to be active on both substrates, due to the similarities between the electronic structures of the two surfaces. However, some differences are detected, which can be related to the higher ionicity and higher energy of $E_{F}$ in TiN, compared to TiC. In particular, the results indicate that a weak adatom-CB coupling is also present.

\section{DISCUSSION AND CONCLUSIONS}

Our previous analysis shows that, from the DOS perspective, our concerted-coupling model is applicable also to adsorption on $\mathrm{TiN}(111)$. In the following, we discuss how this model can be used to account for the essential features of the calculated trends in $E_{\text {ads }}$ 1, 2], which varies in a characteristic pyramid-like manner for the second-period adatoms $\mathrm{B}, \mathrm{C}, \mathrm{N}, \mathrm{O}$, and $\mathrm{F}$, peaking at $\mathrm{O}$, and the third-period adatoms $\mathrm{Al}, \mathrm{Si}, \mathrm{P}, \mathrm{S}$, and $\mathrm{Cl}$, peaking at $\mathrm{S}$, on both $\mathrm{TiC}(111)$ and $\mathrm{TiN}(111)$ [Fig. 3(a)].

Given the strong quenching of the TiSR, a strong contribution to the bonding can be expected to come from the first component of the concerted coupling, the adatom-TiSR coupling. Indeed, the adsorption energy for $\mathrm{O}$ on the TiSR-deficient $\mathrm{TiC}(001)$ surface is $43 \%$ weaker than on TiC(111) [1]. The strong bonding contribution of the TiSR arises from the fact that the antibonding adatom-TiSR solution lies above $E_{F}$, thus remaining empty for all adatoms.
Our calculated $\triangle$ DOS's [Figs. [3 $(\mathrm{b}-\mathrm{k})]$ show that, within each adatom period, the TiSR quenching decreases from group-IV to group-VII adatoms, while it increases from group III to group IV. These trends could indicate an increase in adatom-TiSR coupling strength from group III to group IV and a successive decrease from group IV to group VII. These trends agree with the calculated $E_{\text {ads }}$ trends for $\mathrm{B} \rightarrow \mathrm{C}$, for $\mathrm{Al} \rightarrow \mathrm{Si}$, for $\mathrm{O} \rightarrow \mathrm{F}$, and for $\mathrm{S} \rightarrow \mathrm{Cl}$. Also, the smaller TiSR quenching in period 3 than in period 2 indicates a weaker adatom-TiSR coupling for period 3 , in agreement with our $E_{\text {ads }}$ results.

However, this trend in adatom-TiSR coupling strength disagrees strongly with the calculated $E_{\text {ads }}$ trends for $\mathrm{C}$ $\rightarrow \mathrm{N} \rightarrow \mathrm{O}$ and for $\mathrm{Si} \rightarrow \mathrm{P} \rightarrow \mathrm{S}$. On the other hand, our state-resolved DOS's (see Sec. III.B) show that the second component of the concerted coupling, the adatomXSR couplings, gets successively more active as the energetical overlap between the adlevel and the substrate XSR's increases. As $Z$ increases within each adatom period, the adlevel energy decreases, thus increasing the overlap between the adlevel and the energetical region of the XSR's (that is, the lower half of the substrate UVB). Therefore, the adatom-XSR coupling strengths can be expected to increase as $Z$ increases within each adatom period, providing an explanation for the overall increasing $E_{\text {ads }}$ values from group III to group VI. In addition, this provides further explanation for the stronger chemisorption of the second-period adatoms, compared to the third period, due to the lower free-atom energy, within a given group, of the second-period adatoms.

On the other hand, such a trend is in contrast to the $E_{\text {ads }}$ trends for $\mathrm{O} \rightarrow \mathrm{F}$ and $\mathrm{S} \rightarrow \mathrm{Cl}$. However, $\mathrm{F}$ and $\mathrm{Cl}$ have ionic bonds, as shown by our calculated Bader charges, which yield a charge transfer from the surface $\mathrm{Ti}$ atoms to the $\mathrm{F}$ and $\mathrm{Cl}$ adatoms of $0.75-0.80$ electrons, and by our calculated electron distributions, which show negligible bond charges for $\mathrm{F}$ and $\mathrm{Cl}$ [2]. This implies almost fully occupied outer electron shells for the $\mathrm{F}$ and $\mathrm{Cl}$ adatoms, which can therefore be considered to be almost inert toward further chemical interaction, that is, toward a coupling with the UVB states. Indeed, our $\triangle \mathrm{DOS}$ 's show that the $\mathrm{F}$ and $\mathrm{Cl}$ adlevels do not broaden as significantly as the levels of other adatoms with the same amount of overlap with the UVB region $[c f$., for example, $\mathrm{Cl}$ and $\mathrm{O}$ on $\mathrm{TiN}(111)]$, indicating a weaker adatomUVB coupling. For all the other fcc adatoms, the electron densities show a clear covalent character for the adatomsubstrate bond, with the ionic character increasing successively with increasing $Z$ within each adatom period. This is confirmed by our calculated Bader charges for the adatoms: $-1.07,-1.31,-1.34,-1.20$, and -0.80 for the second-period adatoms $\mathrm{B}, \mathrm{C}, \mathrm{N}, \mathrm{O}$, and $\mathrm{F}$, respectively (corresponding to $21 \%, 33 \%, 45 \%, 60 \%$, and $80 \%$ of their empty outer electron shells) and $-0.40,-0.89$, $-1.08,-1.05$, and -0.74 for the third-period adatoms $\mathrm{Al}, \mathrm{Si}, \mathrm{P}, \mathrm{S}$, and $\mathrm{Cl}$, respectively (corresponding to $8 \%$, $22 \%, 36 \%, 52 \%$, and $74 \%$ of their empty outer electron shells). 

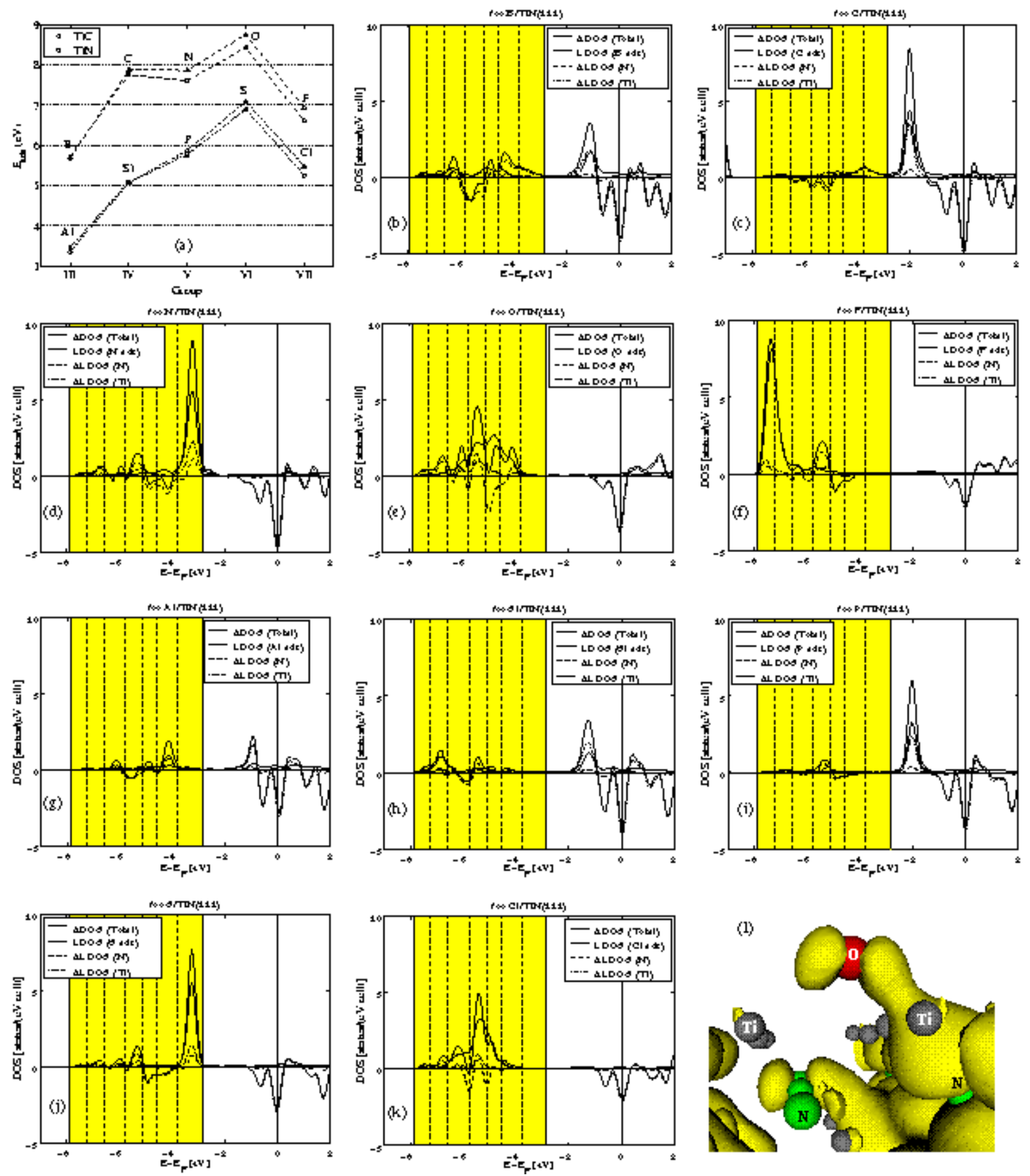

FIG. 3: Adsorption of the second-period adatoms B, C, N, O, and F and of the third-period adatoms Al, Si, P, S, and Cl in fcc site on TiX(111). (a) Calculated trends in adsorption energies $E_{\text {ads }}$ (excerpt from Ref. [2]). (b)-(k) Calculated densities of states (DOS's) for adsorption on TiN(111). Thin line: adatom-projected DOS. Thick line: difference in total DOS ( $\Delta$ DOS) before and after adsorption for the surface TiN bilayer, including adatom. Dashed (dot-dashed) line: $\Delta$ DOS projected onto N (Ti) atoms of the surface bilayer. Shaded area: energetical location of the substrate UVB. Dashed vertical lines: energetical location of the six substrate-UVB peaks. (1) Contour plot of the KS wavefunction at $E=-6.8 \mathrm{eV}$ for the fcc $\mathrm{O}$ adatom on $\mathrm{TiN}(111)$, illustrating a typical bonding adatom-N interaction. 


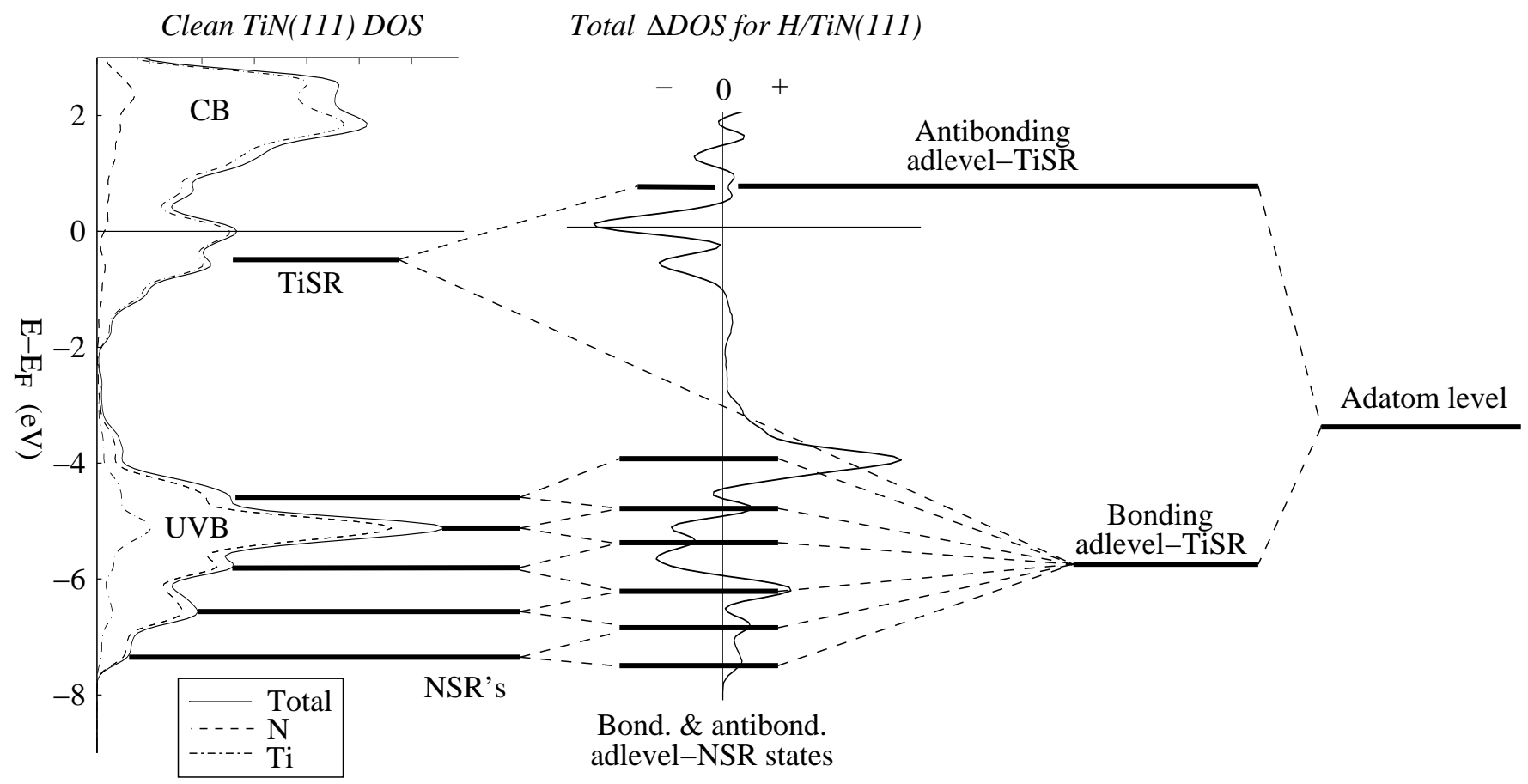

FIG. 4: Schematic diagram of the concerted coupling between the adatom state and the TiN(111) Ti- and N-centered surface resonances (TiSR and NSR's), here illustrated for the case of fcc H/TiN(111). On the left, the DOS for the clean TiN(111) is reproduced. In the middle, our calculated $\triangle \mathrm{DOS}$ for $\mathrm{H} / \mathrm{TiN}(111)$ is shown, illustrating the quenching and appearance of peaks due to adsorption as negative ("-") and positive ("+") peaks, respectively. Horizontal solid lines represent schematically the individual energy levels before and after adsorption.

Thus, the concerted-coupling model, which well describes the calculated DOS results, provides also a way for understanding the main features of the calculated $E_{\text {ads }}$ trends for both TiX(111) surfaces. In particular, our analysis points toward a greater role for the adatomXSR couplings in this understanding than what has been suggested so far in the literature.

Our results show also a small but interesting difference between $\mathrm{TiC}$ and $\mathrm{TiN}$ : for adatoms with low $Z$ (B and $\mathrm{Al}$ ) chemisorption is slightly stronger on $\operatorname{TiN}(111)$, while for adatoms with higher $Z$ it is stronger on $\mathrm{TiC}(111)$. For $\mathrm{B}$ and $\mathrm{Al}$, the adlevels lie above the UVB region. The adatom-XSR contributions to their bonding strengths are therefore negligible and their chemisorption is dominated by the adatom-TiSR coupling. Hence, our $E_{\text {ads }}$ results imply that the bonding contribution from the adatom-TiSR coupling is somewhat stronger on $\mathrm{TiN}(111)$ than on $\mathrm{TiC}(111)$, which is in agreement with our previous observation that the TiSR quenching is stronger on TiN than on TiC. Now, as stated above, as the adlevel energy shifts down and overlaps with the UVB region, the adatom-XSR couplings get successively stronger. However, for the same adlevel shift, this overlap is stronger on $\mathrm{TiC}$ than on TiN, due to the smaller UVBCB energy gap of TiC. Therefore, for the same adatom, the adatom-XSR contributions to the bonding strength are stronger on $\mathrm{TiC}$ than on TiN. As $Z$ increases within each adatom period, this effect grows strong enough to "neutralize" the stronger adatom-TiSR coupling for TiN and invert the chemisorption preference between TiN and TiC.

In summary, the application of our concerted-coupling model, originally proposed for adsorption on $\mathrm{TiC}(111)$, can be generalized to TiN(111). However, the quantitative differences in electronic structures between the two compounds must be taken into account. This shows that adsorption on $\mathrm{TiC}$ and $\mathrm{TiN}$ is more complex and versatile than on, for instance, pure metal surfaces and that the combination of several different mechanisms must be taken into account, giving a potential for variations, very useful in applications.

\section{Acknowledgments}

Valuable discussions with Øyvind Borck are gratefully acknowledged, as is financial support from the Swedish Foundation for Strategic Research via Materials Consortium \#9 and ATOMICS and the Swedish Scientific Council, as well as allocation of computer time at the UNICC facility (Chalmers) and at SNIC (Swedish National Infrastructure for Computing). 
[1] C. Ruberto and B. I. Lundqvist, Phys. Rev. B (submitted). Online version available at http://arxiv.org/abs/cond-mat/0511405

[2] A. Vojvodic, C. Ruberto, and B. I. Lundqvist, Surf. Sci. (submitted). Online version available at http://arxiv.org/abs/cond-mat/0511406

[3] B. Hammer and J. K. Nørskov, in Advances in Catalysis, edited by B. C. Gates and H. Knözinger (Academic Press, San Diego and London 2000), Vol. 45, p. 71.

[4] http://www.camp.dtu.dk/campos

[5] R. F. W. Bader, Chem. Rev. 91 (1991) 893.

[6] C. Oshima, M. Aono, S. Zaima, Y. Shibata, and S. Kawai, J. Less-Common Metals 82 (1981) 69.
[7] These TiSR's have been named surface states in the literature, but we prefer to adopt the proper term surface resonances (SR's), since their energies do not lie in true bulk band gaps [see Figs. 11 (a-b)].

[8] M. Tsukada and T. Hoshino, J. Phys. Soc. Jpn. 51 (1982) 2562.

[9] D. M. Newns, Phys. Rev. 178 (1969) 1123.

[10] D. Spanjaard and M.C. Desjonquères, in Interaction of Atoms and Molecules with Solid Surfaces, edited by V. Bortolani, N.H. March, and M.P. Tosi (Plenum Press, New York and London 1990), Chap. 9. 